\title{
Stochastic Multiplicative DEA Model for Measuring the Efficiency and Ranking of DMUs under VRS Technology
}

\author{
H. Dibachi ${ }^{1 *}$, M. H. Behzadi ${ }^{1}$ and M. Izadikhah ${ }^{2}$ \\ 'Department of Statistics, Science and Research Branch, Islamic Azad University, Tehran, Iran; \\ h-dibachi@iau-arak.ac.ir, behzadi@srbiau.ac.ir \\ ${ }^{2}$ Department of Mathematics, Arak-Branch, Islamic Azad University, Arak, Iran; \\ m-izadikhah@iau-arak.ac.ir
}

\begin{abstract}
One class of models introduced in data envelopment analysis is called multiplicative model. In this paper, the extension of multiplicative data envelopment analysis(MDEA) model under variable return to scale(VRS) technology in the presence of stochastic data was focused on. The log-normal distribution is a family of probability density functions that is frequently used in practical situations. Therefore, in the present study, a stochastic MDEA model under VRS technology was proposed for measuring the stochastic $\alpha$ - efficiency of decision making units (DMUs) with inputs and outputs having log-normal distributions. Moreover, stochastic super-efficiency model was proposed for ranking stochastic $\alpha$ - efficient DMUs in stochastic MDEA model. Finally, an example of the system reliability was presented for 12 different brands of computers to demonstrate our proposed modeling idea and its efficiency.
\end{abstract}

Keywords: Log-normal Distribution, Multiplicative Data Envelopment Analysis(MDEA), Stochastic MDEA, Stochastic $\alpha-$ efficiency, Stochastic Super-efficiency, System Reliability

\section{Introduction}

Data envelopment analtsis (DEA), as initiated and developed by Charnes ${ }^{1}$ is a non-parametric method for identifying efficient production frontiers and evaluating the relative efficiency of decision making units (DMUs) with similar quantitative characteristics. This is reflected by the assumption that each DMU uses the same set of inputs to produce the same set of outputs.

One class of models introduced in DEA is called multiplicative data envelopment analysis (MDEA) model, as initiated and developed by Banker and Maindiratta ${ }^{2}$, the piecewise linear frontiers usually employed in DEA are replaced by a frontier that is piecewise Cobb-Douglas and introduced also a model to identify the most productive scale size pattern. Banker. ${ }^{3}$ presented a two-stage method for the identification of returns to scale in MDEA model. In the BCC model the convexity postulate permits increasing, constant or decreasing returns to scale in different regions of the production function. However, this also requires the marginal products (see Menger ${ }^{4}$ ), for a comparison of returns to scale and rate of change of marginal product) to be nonincreasing. This restriction in the BCC approach may not be appropriate for production technologies where the production function is nonconcave in some regions and the production possibility set is not convex. To allow for such situations, replace the (ordinary) convexity postulate of BCC by "geometric" convexity to interpolate between observed production possibilities. This implies that the piecewise linear frontiers usually employed in DEA are replaced by a frontier that is piecewise Cobb-Douglas. If in an empirical application there are a priori reasons to believe that marginal products are increasing in some regions, then the log-linear model is the appropriate DEA model for the analysis.

In cases in which several DMUs have the same efficiency score of 1 , a standard DEA approach is not able to discriminate amongst this DMUs. There are several approaches in the literature to rank efficient DMUs in DEA. Adler et al. ${ }^{5}$ classified these approaches into six streams. Perhaps super-efficiency is the most well known, most widely applied and researched ranking method

${ }^{*}$ Author for correspondence 
in DEA. The idea of super-efficiency as developed by Andersen and Petersen ${ }^{6}$.

The stochastic data envelopment analysis (SDEA) approach was developed by considering the value of inputs and outputs as random variables. Olesen and Petersen ${ }^{7}$ developed a chance-constrained DEA model which used the piecewise linear envelopments of confidence regions for use with stochastic multiple inputs and multiple outputs. Cooper. ${ }^{8}$ developed a "joint chance-constrained" DEA model to naturally generalize "Pareto-Koopman's Efficiency" to stochastic situations. Huang ${ }^{9}$ utilized this joint chance-constrained concept to discuss general dominance structures in the stochastic situations.

Almost, all of the previous works in SDEA have been used the stochastic data when the inputs and outputs having normal distributions. As far as we know stochastic MDEA models have not already been used. when dealing with failure and repair mechanisms in general, the most suitable and applied distribution is the log-normal distribution. Therefore, in this paper, we propose the stochastic MDEA model under VRS technology and stochastic super-efficiency for measuring the efficiency and ranking of systems. We consider these systems as DMUs with the inputs and outputs having log-normal distributions where inputs and outputs are stochastic failure times and stochastic repair times, respectively. The paper unfolds as follows:

Some basic concepts in statistics and deterministic MDEA model will be introduced in the next section. Section 3 addresses the proposed method for introducing the stochastic MDEA model. Section 4 addresses the stochastic super-efficiency proposed model for ranking of DMUs in stochastic MDEA model. A brief discuss about the proposed models and an applied example are given in section 5. Conclusions will appear in section 6.

\section{Preliminaries}

In this section, we recall some basic concepts and results which will be used through the paper.

\subsection{Log-normal Distribution}

The log-normal distribution is a family of probability density functions that is frequently used in practical situations. In system reliability analysis, failure time and repair time a system is often distributed log-normally.

Definition 2.1 A random variable $X$ is said to have log-normal distribution if its probability density function $(P D F)$ is given as follows:

$$
f_{X}(x)= \begin{cases}\frac{1}{x \sqrt{2 \pi \sigma^{2}}} e^{-\frac{1}{2 \sigma^{2}}(\ln x-\mu)^{2}} & ; x>0 \\ 0 & ; \text { o.w }\end{cases}
$$

We will use the notation $X \sim L N\left(\mu, \sigma^{2}\right)$ to denote a random variable $\mathrm{X}$ having the log-normal distribution with parameters $\sigma>0$ and $\mu \in R$ where $\mu=E(\operatorname{LnX})$ and $\sigma^{2}=\operatorname{Var}(\operatorname{LnX})$.

\section{REMARK 2.1}

If $X \sim L N\left(\mu, \sigma^{2}\right)$, then $Y=\operatorname{LnX}$ having the normal distribution with scale parameter $\sigma>0$ and location parameter $\mu \in R$ where is denoted by notation $Y \sim N\left(\mu, \sigma^{2}\right)$. Thus, $P D F$ random variable $Y$ is given as follows:

$$
f_{Y}(y)=\frac{1}{\sqrt{2 \pi \sigma^{2}}} e^{-\frac{1}{2 \sigma^{2}}(y-\mu)^{2}} ; y \in R
$$

The corresponding cumulative distribution function (CDF) has the following form:

$$
F_{Y}(y)=\int_{-\infty}^{y} \frac{1}{\sqrt{2 \pi \sigma^{2}}} e^{-\frac{1}{2 \sigma^{2}}(t-\mu)^{2}} d t
$$

Note that if $Y \sim N(0,1)$ then $f_{Y}(y)$ is called standard normal distribution and $f_{Y}(y)$ is denoted by $\Phi(y)$ and $\Phi^{-1}$, its inverse, is the so-called fractile function. Specially, $\Phi^{-1}(0.5)=0, \Phi^{-1}(0.1)=-1.28$, and $\Phi^{-1}(0.67)=0.44$.

\subsection{System Reliability}

A system contains one or several subsystems of components interconnected so that the system is able to perform of number of required functions. The reliability of the system denotes the relationship between the systems required performance and its achieved performance. The probabilistic method of the system's reliability deals with the uncertainty of this relation. To discuss a component's characteristics in terms of reliability there are several functions that can be utilized. The failure rate function, $w(t)$ illustrates the components tendency to fail, failures per time unit, for $t$ $\geq 0$. However, the instantaneous failure rate at the time $t_{0}$ for functional rate components is called $\gamma=w\left(t_{0}\right)$, the corresponding instantaneous repair rate for faulted items is called $\mu$. In order to comprehend a component's stochastic behaviour concerning its uptime, functional, and downtime, faulted, the components probabilistic behaviour can be represented using a probability density function. In reliability analysis, failure time and repair time a system is often distributed log-normally. Therefore, in this paper used from the log-normal distribution. 


\subsection{Output-Oriented BCC Model}

One of the basic DEA model for evaluating DMUs is the output-oriented BCC model where introduced by Banker ${ }^{10}$. They omitted the ray unboundedness postulate from the CCR postulates and deduced the following production possibility set:

$T_{v}=\left\{(X, Y) \mid X \geq \sum_{j=1}^{n} \lambda_{j} X_{j} \& Y \leq \sum_{j=1}^{n} \lambda_{j} Y_{j} \& \sum_{j=1}^{n} \lambda_{j}=1 \& \lambda_{j} \geq 0\right\}$

Where $X_{j}=\left(x_{1 j}, x_{2 j}, \ldots, x_{m j}\right)$ and $Y_{j}=\left(y_{1 j}, y_{2 j}, \ldots, y_{s j}\right)$ are the input and output vectors of $D M U$, respectively. Moreover, $x_{i j} \geq 0$ and $y_{r j} \geq 0$ for each $r \in\{1,2, \ldots, s\}$, $j \in\{1,2, \ldots, n\}$, and $i \in\{1,2, \ldots, m\}$. The BCC-efficiency of a specific $D M U_{o}$ under set $T_{v}$ is obtained by solving the following model:

$$
\begin{aligned}
& \varphi_{o}^{*}=\max \quad \varphi_{o} \\
& \text { s.t. } \\
& \sum_{j=1}^{n} \lambda_{j} y_{r j}-s_{r}^{+}=\varphi_{o} y_{r o}, \quad r=1, \ldots, s, \\
& \sum_{j=1}^{n} \lambda_{j} x_{i j}+s_{i}^{-}=x_{i o}, \quad i=1, \ldots, m, \\
& \sum_{j=1}^{n} \lambda_{j}=1 \\
& \lambda_{j} \geq 0 \text {, } \\
& s_{i}^{-} \geq 0 \text {, } \\
& s_{r}^{+} \geq 0 \text {, } \\
& j=1, \ldots, n, \\
& i=1, \ldots, m \text {, } \\
& r=1, \ldots, s,
\end{aligned}
$$

\section{DEFINITION 2.2}

(BCC-efficient) $D M U_{o}$ is said to be BCC-efficient if and only if the following two conditions are both satisfied:

\section{i. $\varphi_{o}^{*}=1$}

ii. All slack variables are zero in the alternative optimal solution.

\subsection{Output-Oriented MDEA Model}

Suppose that there are $\mathrm{n}$ DMUs, where each $D M U_{j}$ $(\mathrm{j}=1, \ldots, \mathrm{n})$ uses $\mathrm{m}$ different inputs, $x_{i j}>0(\mathrm{i}=1, \ldots, \mathrm{m})$, to producesdifferentoutputs, $y_{r j}>0(\mathrm{r}=1, \ldots, \mathrm{s})$ and supposealso that the data set is deterministic. Therefore for each $D M U_{j}$, let $X_{j}=\left(x_{1 j}, x_{2 j}, \ldots, x_{m j}\right)$ and $Y_{j}=\left(y_{1 j}, y_{2 j}, \ldots, y_{s j}\right)$ are the input and output vectors of $D M U_{j}$, respectively. One of the basic multiplicative data envelopment analysis (MDEA) models used to evaluate DMUs is the output-oriented MDEA model under VRS technology. Banker and Maindiratta ${ }^{2}$ replaced the (ordinary) convexity postulate of BCC by "geometric" convexity, and introduced the following production possibility set (PPS):

$$
T_{v}^{\prime}=\left\{(X, Y) \mid X \geq \prod_{j=1}^{n} X_{j}^{\lambda_{j}} \& Y \leq \prod_{j=1}^{n} Y_{j}^{\lambda_{j}} \& \sum_{j=1}^{n} \lambda_{j}=1 \& \lambda_{j} \geq 0\right\}
$$

Therefore, output-oriented MDEA model under VRS technology for evaluating $D M U_{o}$ is given by the following model:

$$
\begin{array}{ll}
\varphi_{o}^{*}=\max & \varphi_{o} \\
\text { s.t. } & \\
& \prod_{j=1}^{n} y_{r j}^{\lambda_{j}} \geq \varphi_{o} y_{r o}, \quad r=1, \ldots, s, \\
& \prod_{j=1}^{n} x_{i j}^{\lambda_{j}} \leq x_{i o}, \quad i=1, \ldots, m, \\
& \sum_{j=1}^{n} \lambda_{j}=1, \\
& \lambda_{j} \geq 0,
\end{array}
$$

Now, by taking the natural logarithm of both sides, in the first and second constraint we have:

$$
\begin{array}{lll}
\varphi_{o}^{*}=\max & \varphi_{o} \\
\text { s.t. } & \\
& \sum_{j=1}^{n} \lambda_{j} \operatorname{Ln} y_{r j} \geq \operatorname{Ln} \varphi_{o}+\operatorname{Ln} y_{r o}, \quad r=1, \ldots, s, \\
& \sum_{j=1}^{n} \lambda_{j} \operatorname{Ln} x_{i j} \leq \operatorname{Lnx} x_{i o}, & \\
& \sum_{j=1}^{n} \lambda_{j}=1, & \\
& \lambda_{j} \geq 0, &
\end{array}
$$

Alternatively,

$$
\begin{aligned}
& \varphi_{o}^{*}=\max \quad \varphi_{o} \\
& \text { s.t. } \\
& \sum_{j=1}^{n} \lambda_{j} \operatorname{Ln} y_{r j}-s_{r}^{+}=\operatorname{Ln} \varphi_{o}+\operatorname{Ln} y_{r o}, \quad r=1, \ldots, s, \\
& \sum_{j=1}^{n} \lambda_{j} \operatorname{Ln} x_{i j}+s_{i}^{-}=\operatorname{Ln} x_{i o}, \quad i=1, \ldots, m, \\
& \sum_{j=1}^{n} \lambda_{j}=1 \\
& \lambda_{j} \geq 0, \quad j=1, \ldots, n, \\
& s_{i}^{-} \geq 0, \quad i=1, \ldots, m, \\
& s_{r}^{+} \geq 0, \quad r=1, \ldots, s,
\end{aligned}
$$


Where $s_{i}^{-} \geq 0$ and $s_{r}^{+} \geq 0$ represent slacks.

Definition 2.3 (MDEA-efficient) $\mathrm{DMU}_{o}$ is said to be MDEA-efficient if and only if the following two conditions are both satisfied:

i. $\varphi_{o}^{*}=1$

ii. All slack variables are zero in the alternative optimal solution.

\section{Stochastic MDEA Model}

In this section, we present the stochastic MDEA Model. For each $D M U_{j}$, let $X_{j}=\left(X_{1 j}, X_{2 j}, \ldots, X_{m j}\right.$ and $Y_{j}=\left(Y_{1 j}, Y_{2 j}, \ldots, Y_{s j}\right.$ are the input and output random vectors of $D M U_{j}$, respectively. Moreover, suppose that $X_{i j}>0$ and $Y_{r j}>0$. If $X_{i j} \sim L N\left(\mu_{i j}, \sigma_{i j}^{2}\right)$ and $Y_{r j} \sim L N\left(\gamma_{r j}, \tau_{r j}^{2}\right)$ then by $\operatorname{Remark}(2.1) \quad \operatorname{Ln} X_{i j}=\tilde{X}_{i j} \sim N\left(\mu_{i j}, \sigma_{i j}^{2}\right)$ and $\operatorname{Ln} Y_{r j}=\tilde{Y}_{r j} \sim N\left(\gamma_{r j}, \tau_{r j}^{2}\right)$. Now, by using models (7) and (8) the stochastic output-oriented MDEA model can be obtained as follows:

$$
\begin{aligned}
& \varphi_{o}^{*}(\alpha)=\max \quad \varphi_{o} \\
& \text { s.t. } \\
& P\left(\prod_{j=1}^{n} Y_{r j}^{\lambda_{j}} \geq \varphi_{o} Y_{r o}\right) \geq 1-\alpha, \quad r=1, \ldots, s, \\
& P\left(\prod_{j=1}^{n} X_{i j}^{\lambda_{j}} \leq X_{i o}\right) \geq 1-\alpha \quad i=1, \ldots, m, \\
& \sum_{j=1}^{n} \lambda_{j}=1 \\
& \lambda_{j} \geq 0 \text {, } \\
& j=1, \ldots, n \text {, }
\end{aligned}
$$

Alternatively,

$$
\begin{aligned}
& \varphi_{o}^{*}(\alpha)=\max \quad \varphi_{o} \\
& \text { s.t. } \\
& P\left(\sum_{j=1}^{n} \lambda_{j} \tilde{Y}_{r j} \geq \operatorname{Ln} \varphi_{o}+\tilde{Y}_{r o}\right) \geq 1-\alpha, \quad r=1, \ldots, s, \\
& P\left(\sum_{j=1}^{n} \lambda_{j} \tilde{X}_{i j} \leq \tilde{X}_{i o}\right) \geq 1-\alpha, \quad i=1, \ldots, m, \\
& \sum_{j=1}^{n} \lambda_{j}=1 \\
& \lambda_{j} \geq 0 \text {, } \\
& j=1, \ldots, n,
\end{aligned}
$$

where $\alpha$ is a predetermined number between 0 and 1 which specifies the significance level and $\mathrm{P}$ means "Probability Measure".

\subsection{Deterministic Equivalent}

If $\tilde{X}_{i j} \sim N\left(\mu_{i j}, \sigma_{i j}^{2}\right)$ and $\tilde{Y}_{r j} \sim N\left(\gamma_{r j}, \tau_{r j}^{2}\right)$ then for all $r \in\{1,2, \ldots, s\}, o \in\{1,2, \ldots, n\}$, and $i \in\{1,2, \ldots, m\}$ we have:

$$
\begin{aligned}
& \sigma_{i}^{2}(\lambda)=\operatorname{Var}\left(\sum_{j=1}^{n} \lambda_{j} \tilde{X}_{i j}-\tilde{X}_{i 0}\right) \\
& =\sum_{\substack{k=1 \\
k \neq 1}}^{n} \sum_{\substack{j=1 \\
j \neq 0}}^{n} \lambda_{j} \lambda_{k} \operatorname{Cov}\left(\tilde{X}_{i k}, \tilde{X}_{i j}\right) \\
& +2\left(\lambda_{0}-1\right) \sum_{\substack{j=1 \\
j \neq 0}}^{n} \lambda_{j} \operatorname{Cov}\left(\tilde{X}_{i j}, \tilde{X}_{i 0}\right)+\left(\lambda_{0}-1\right)^{2} \sigma_{i 0}^{2}
\end{aligned}
$$

Similarly,

$$
\begin{aligned}
\tau_{r}^{2}(\lambda)= & \operatorname{Var}\left(\sum_{j=1}^{n} \lambda_{j} \tilde{Y}_{r j}-\tilde{Y}_{r 0}-\operatorname{Ln} \varphi_{0}\right) \\
= & \operatorname{Var}\left(\sum_{j=1}^{n} \lambda_{j} \tilde{Y}_{r j}-\tilde{Y}_{r 0}\right)=\sum_{\substack{k=1 \\
k \neq 0}}^{n} \sum_{\substack{j=1 \\
j \neq 0}}^{n} \lambda_{j} \lambda_{k} \operatorname{Cov}\left(\tilde{Y}_{r k}, \tilde{Y}_{r j}\right) \\
& +2\left(\lambda_{0}-1\right) \sum_{\substack{j=1 \\
j \neq 0}}^{n} \lambda_{j} \operatorname{Cov}\left(\tilde{Y}_{r j}, \tilde{Y}_{r 0}\right)+\left(\lambda_{0}-1\right)^{2} \tau_{r 0}^{2}
\end{aligned}
$$

\section{THEOREM 3.1}

Deterministic equivalent of stochastic MDEA model (11) is as follows:

$$
\varphi_{o}^{*}(\alpha)=\max \quad \varphi_{o}
$$

s.t.

$$
\begin{array}{ll}
\sum_{j=1}^{n} \lambda_{j} \gamma_{r j}-s_{r}^{+}-\gamma_{r o}+\tau_{r}(\lambda) \Phi^{-1}(\alpha)=\operatorname{Ln} \varphi_{o}, & r=1, \ldots, s, \\
\sum_{j=1}^{n} \lambda_{j} \mu_{i j}+s_{i}^{-}-\sigma_{i}(\lambda) \Phi^{-1}(\alpha)=\mu_{i o}, & i=1, \ldots, m, \\
\sum_{j=1}^{n} \lambda_{j}=1, & \\
s_{i}^{-} \geq 0, & \\
s_{r}^{+} \geq 0, & i=1, \ldots, m, \\
\lambda_{j} \geq 0, & r=1, \ldots, s, \\
& j=1, \ldots, n
\end{array}
$$


Proof: From the first constraint in model (11) and statement (13) we have:

$P\left(\sum_{j=1}^{n} \lambda_{j} \tilde{Y}_{r j} \geq \operatorname{Ln} \varphi_{o}+\tilde{Y}_{r o}\right) \geq 1-\alpha \Leftrightarrow$
$P\left(\begin{array}{c}\frac{\sum_{j=1}^{n} \lambda_{j} \tilde{Y}_{r j}-\tilde{Y}_{r o}-\operatorname{Ln} \varphi_{o}-\left(\sum_{j=1}^{n} \lambda_{j} \gamma_{r j}-\gamma_{r o}-\operatorname{Ln} \varphi_{o}\right)}{\tau_{r}(\lambda)} \\ \geq \frac{-\sum_{j=1}^{n} \lambda_{j} \gamma_{r j}+\gamma_{r o}+\operatorname{Ln} \varphi_{o}}{\tau_{r}(\lambda)}\end{array}\right) \geq 1-\alpha \Leftrightarrow$

$P\left(Z \geq \frac{-\sum_{j=1}^{n} \lambda_{j} \gamma_{r j}+\gamma_{r o}+\operatorname{Ln} \varphi_{o}}{\tau_{r}(\lambda)}\right) \geq 1-\alpha \Leftrightarrow$

$P\left(Z \leq \frac{-\sum_{j=1}^{n} \lambda_{j} \gamma_{r j}+\gamma_{r o}+\operatorname{Ln} \varphi_{o}}{\tau_{r}(\lambda)}\right) \leq \alpha \Leftrightarrow$

$P\left(Z \leq \frac{-\sum_{j=1}^{n} \lambda_{j} \gamma_{r j}+\gamma_{r o}+\operatorname{Ln} \varphi_{o}+s_{r}^{+}}{\tau_{r}(\lambda)}\right)=\alpha \Leftrightarrow$

$\Phi\left(\frac{-\sum_{j=1}^{n} \lambda_{j} \gamma_{r j}+\gamma_{r o}+\operatorname{Ln} \varphi_{o}+s_{r}^{+}}{\tau_{r}(\lambda)}\right)=\alpha \Leftrightarrow$

$\Phi^{-1}(\alpha)=\frac{-\sum_{j=1}^{n} \lambda_{j} \gamma_{r j}+\gamma_{r o}+\operatorname{Ln} \varphi_{o}+s_{r}^{+}}{\tau_{r}(\lambda)} \Leftrightarrow$

$\sum_{j=1}^{n} \lambda_{j} \gamma_{r j}-s_{r}^{+}-\gamma_{r o}+\tau_{r}(\lambda) \Phi^{-1}(\alpha)=\operatorname{Ln} \varphi_{o}$
Similarly, from the second constraint in model (11) and statement (12) we have:

$$
\begin{aligned}
& P\left(\sum_{j=1}^{n} \lambda_{j} \tilde{X}_{i j} \leq \tilde{X}_{i o}\right) \geq 1-\alpha \Leftrightarrow \\
& P\left(\frac{\sum_{j=1}^{n} \lambda_{j} \tilde{X}_{i j}-\tilde{X}_{i o}-\left(\sum_{j=1}^{n} \lambda_{j} \mu_{i j}-\mu_{i o}\right)}{\sigma_{i}(\lambda)} \leq \frac{-\sum_{j=1}^{n} \lambda_{j} \mu_{i j}+\mu_{i o}}{\sigma_{i}(\lambda)}\right) \geq 1-\alpha \Leftrightarrow
\end{aligned}
$$$$
P\left(Z \leq \frac{-\sum_{j=1}^{n} \lambda_{j} \mu_{i j}+\mu_{i o}}{\sigma_{i}(\lambda)}\right) \geq 1-\alpha \Leftrightarrow
$$

$P\left(Z \leq \frac{\sum_{j=1}^{n} \lambda_{j} \mu_{i j}-\mu_{i o}}{\sigma_{i}(\lambda)}\right) \leq \alpha \Leftrightarrow$

$P\left(Z \leq \frac{\sum_{j=1}^{n} \lambda_{j} \mu_{i j}-\mu_{i o}+s_{i}^{-}}{\sigma_{i}(\lambda)}\right)=\alpha \Leftrightarrow$

$\Phi\left(\frac{\sum_{j=1}^{n} \lambda_{j} \mu_{i j}-\mu_{i o}+s_{i}^{-}}{\sigma_{i}(\lambda)}\right)=\alpha \Leftrightarrow$

$\Phi^{-1}(\alpha)=\frac{\sum_{j=1}^{n} \lambda_{j} \mu_{i j}-\mu_{i o}+s_{i}^{-}}{\sigma_{i}(\lambda)} \Leftrightarrow$

$\sum_{j=1}^{n} \lambda_{j} \mu_{i j}+s_{i}^{-}-\sigma_{i}(\lambda) \Phi^{-1}(\alpha)=\mu_{i o}$

Thus, by (15) and (16), the deterministic model is completely specified. Therefor, $\varphi_{o}^{*}(\alpha), s_{i}^{-*}$, and $s_{i}^{+*}$ can be determined by solving model (14).

\section{DEFINITION 3.1}

$D M U_{o}$ is stochastic $\alpha-e$ efficient if and only if the following two conditions are both satisfied:

i. $\varphi_{o}^{*}(\alpha)=1$

ii. All slack variables are zero in the alternative optimal solution. 
Otherwise, $D M U_{o}$ is called stochastic $\alpha$ - inefficient. For the sake of simplicity, we denote them by $\alpha$ - efficient and $\alpha$ - inefficient, respectively.

In order to determine the stochastic $\alpha$ - efficiency we consider the following theorem:

Theorem 3.2 For $\alpha=0.5$. The inefficiency vs. efficiency classification of $D M U_{o}$ in output-oriented MDEA model (9) is the same as in stochastic MDEA model (14).

Proof: If $\alpha=0.5$ then $\Phi^{-1}(0.5)=0$. Thus, the inefficiency vs. efficiency classification of $D M U_{o}$ in deterministic MDEA model (9) is the same as in stochastic MDEA model (14).

\section{Stochastic Super-Efficiency Model}

One of the basic models for ranking DMUs is the "superefficiency" model where introduced by Anderson and Peterson $^{2}$. The efficiency scores from these models are obtained by eliminating the data on the $D M U_{o}$ to be evaluated from the solution set. For the input model this can result in values which are regarded as according $D M U_{o}$ the status of being "super-efficient". These values are used to rank the DMUs and thereby eliminate some (but not all) of the ties that occur for efficient DMUs. Now, by using model (11) we propose the stochastic super-efficiency for stochastic MDEA model (10)as follows :

$$
\begin{aligned}
& P\left(\sum_{\substack{j=1 \\
j \neq 0}}^{n} \lambda_{j} \tilde{Y}_{r j} \geq \operatorname{Ln} \varphi_{0}+\tilde{Y}_{r 0}\right) \geq 1-\alpha, \quad r=1, \ldots, s, \\
& P\left(\sum_{\substack{j=1 \\
j \neq 0}}^{n} \lambda_{j} \tilde{X}_{i j} \leq \tilde{X}_{i 0}\right) \geq 1-\alpha, \quad i=1, \ldots, m, \\
& \sum_{\substack{j=1 \\
j \neq 0}}^{n} \lambda_{j}=1, \\
& \lambda_{j} \geq 0,
\end{aligned}
$$

In a similar manner to the proof of Theorem (3.1) the deterministic equivalent of stochastic super-efficiency model (17) is specified as follows:

$\varphi_{o}^{*}(\alpha)=\max \varphi_{o}$

s.t.

$\left(\sum_{\substack{j=1 \\ j \neq 0}}^{n} \lambda_{j} \gamma_{r j}-S_{r}^{+}-\gamma_{r 0}+\tau_{r}(\lambda) \Phi^{-1}(\alpha)=\operatorname{Ln} \varphi_{0},\right) r=1, \ldots, s$,
$\left(\sum_{\substack{j=1 \\ j \neq 0}}^{n} \lambda_{j} \mu_{i j}+S_{r}^{-}-\sigma_{i}(\lambda) \Phi^{-1}(\alpha)=\mu_{i 0}\right.$,

$\sum_{\substack{j=1 \\ j \neq 0}}^{n} \lambda_{j}=1$,

$S_{i}^{-} \geq 0$,

$i=1, \ldots, m$,

$S_{r}^{+} \geq 0$,

$r=1, \ldots, s$,

$\lambda_{i} \geq 0$,

$j=1, \ldots, n$

Note that in the above model we have:

$\begin{aligned} \sigma_{i}^{2}(\lambda) & =\operatorname{Var}\left(\sum_{\substack{j=1 \\ j \neq 0}}^{n} \lambda_{j} \tilde{X}_{i j}-\tilde{X}_{i 0}\right) \\ & =\sum_{\substack{k=1 \\ k \neq 1}}^{n} \sum_{\substack{j=1 \\ j \neq 0}}^{n} \lambda_{j} \lambda_{k} \operatorname{Cov}\left(\tilde{X}_{i k}, \tilde{X}_{i j}\right)-2 \sum_{\substack{j=1 \\ j \neq 0}}^{n} \lambda_{j} \operatorname{Cov}\left(\tilde{X}_{i j}, \tilde{X}_{i 0}\right)+\sigma_{i 0}^{2}\end{aligned}$

$$
\begin{aligned}
\tau_{r}^{2}(\lambda) & =\operatorname{Var}\left(\sum_{\substack{j=1 \\
j \neq 0}}^{n} \lambda_{j} \tilde{Y}_{r j}-\tilde{Y}_{r 0}-\operatorname{Ln} \varphi_{0}\right)=\operatorname{Var}\left(\sum_{\substack{j=1 \\
j \neq 0}}^{n} \lambda_{j} \tilde{Y}_{r j}-\tilde{Y}_{r 0}\right) \\
& =\sum_{\substack{k=1 \\
k \neq 0}}^{n} \sum_{\substack{j=1 \\
j \neq 0}}^{n} \lambda_{j} \lambda_{k} \operatorname{Cov}\left(\tilde{Y}_{r k}, \tilde{Y}_{r j}\right)-2 \sum_{\substack{j=1 \\
j \neq 0}}^{n} \lambda_{j} \operatorname{Cov}\left(\tilde{Y}_{r j}, \tilde{Y}_{r 0}\right)+\tau_{r 0}^{2}
\end{aligned}
$$

Stochastic super-efficiency model (17) computes the score of the DMU under evaluation with removing it from constraints. 


\section{DEFINITION 4.1}

$D M U_{o}$ is stochastic super $\alpha-$ efficient if $0<\varphi_{o}^{*}(\alpha)<1$.

Moreover, if $\varphi_{o}^{*}(\alpha)>1$ then $D M U_{o}$ is called stochastic super $\alpha$-inefficient. For the sake of simplicity, we denote them by super $\alpha$-efficient and super $\alpha$-inefficient, respectively.

\section{Application}

In this section, an example of system reliability is presented to demonstrate the modeling idea and the effectiveness of the proposed method.

\subsection{Practical Example of System Reliability}

We apply the proposed stochastic MDEA methodology and stochastic super-efficiency for measuring the efficiency and ranking of 12 systems. We consider this systems as DMUs, and denote them by $D M U_{j},(j=1,2, \ldots, 12)$. Every $D M U_{j}$ is composed of 2 components which have the random length of time until failure. Suppose that $Y_{r j}, r=1,2$ are the random failure time of component $r$ of $D M U_{j}$ where have the log-normal distribution with parameters $\tau_{r j}^{2}$ and $\gamma_{r j}$ which are denoted with $Y_{r j} \sim L N\left(\gamma_{r j}, \tau_{r j}^{2}\right)$. When a component fails it undergoes repair. Suppose that $X_{i j}, i=1,2$ are the random repair time of component i of $D M U_{j}$ where have the log-normal distribution with parameters $\sigma_{i j}^{2}$ and $\mu_{i j}$ which are denoted with $X_{i j} \sim L N\left(\mu_{i j}, \sigma_{i j}^{2}\right)$. Let us consider 12 different brands of computers(for example, Acer, Apple, Compaq, Dell, Emachines, Everex, Hp, Ibm/Lenovo, Panasonic, Sony, Toshiba, and Gateway) and consider also two principal components(or items) of computers. Thus, by solving models(14) and (18) can be obtain the stochastic $\alpha$ - efficiency of computers (i.e. DMUs) and its rank. The labels of inputs and outputs are as Table 1.

The data set for this example is shown in Table 2 .

We run models (14) and (18) by means of GAMS software for all $\alpha \in\{0.1,0.5,0.67\}$ and the results are shown in Table 3.

There are a lot of number of the DMUs, which are $\alpha$-efficient thus, by using stochastic super-efficiency are

Table 1. The labels of inputs and outputs.

\begin{tabular}{ll}
\hline Input1 & $\begin{array}{l}\text { The random repair time of the first } \\
\text { component of computer }\end{array}$ \\
Input2 & $\begin{array}{l}\text { The random repair time of the second } \\
\text { component of computer }\end{array}$ \\
Output1 & $\begin{array}{l}\text { The random length of time until failure of } \\
\text { the first component of computer }\end{array}$ \\
Output2 & $\begin{array}{l}\text { The random length of time until failure of } \\
\text { the second component of computer }\end{array}$ \\
\hline
\end{tabular}

Table 2. The data set of practical example.

\begin{tabular}{llllll}
\hline $\mathbf{j}$ & \multicolumn{1}{c}{$D U_{j}$} & Input 1 & Input 2 & Output 1 & Output 2 \\
\hline 1 & Acer & $X_{11} \sim L N(20,25)$ & $X_{21} \sim L N(25,16)$ & $Y_{11} \sim L N(1000,100)$ & $Y_{21} \sim L N(900,400$ \\
2 & Panasonic & $X_{12} \sim L N(15,4$ & $X_{22} \sim L N(23,18)$ & $Y_{12} \sim L N(800,200)$ & $Y_{22} \sim L N(950,300)$ \\
3 & Sony & $X_{13} \sim L N(10,4)$ & $X_{23} \sim L N(9,9)$ & $Y_{13} \sim L N(950,400)$ & $Y_{23} \sim L N(500,450)$ \\
4 & Apple & $X_{14} \sim L N(18,8)$ & $X_{24} \sim L N(10,8)$ & $Y_{14} \sim L N(850,500)$ & $Y_{24} \sim L N(550,430$ \\
5 & Hp & $X_{15} \sim L N(17,6)$ & $X_{25} \sim L N(18,7)$ & $Y_{15} \sim L N(980,550)$ & $Y_{25} \sim L N(800,100)$ \\
6 & Everex & $X_{16} \sim L N(16,4)$ & $X_{26} \sim L N(19,15)$ & $Y_{16} \sim L N(700,520)$ & $Y_{26} \sim L N(600,250)$ \\
7 & Emachines & $X_{17} \sim L N(11,9)$ & $X_{27} \sim L N(20,14)$ & $Y_{17} \sim L N(750,700)$ & $Y_{27} \sim L N(650,230$ \\
8 & Compaq & $X_{18} \sim L N(19,20$ & $X_{28} \sim L N(17,4)$ & $Y_{18} \sim L N(850,350)$ & $Y_{28} \sim L N(830,450)$ \\
9 & Dell & $X_{19} \sim L N(12,10)$ & $X_{29} \sim L N(15,17)$ & $Y_{19} \sim L N(600,150)$ & $Y_{29} \sim L N(580,160)$ \\
10 & Ibm/Lenovo & $X_{110} \sim L N(13,5)$ & $X_{210} \sim L N(10,12)$ & $Y_{110} \sim L N(970,300)$ & $Y_{210} \sim L N(560,400)$ \\
11 & Toshiba & $X_{111} \sim L N(16,6)$ & $X_{211} \sim L N(22,16)$ & $Y_{111} \sim L N(780,110)$ & $Y_{211} \sim L N(700,350)$ \\
12 & Gateway & $X_{112} \sim L N(9,4)$ & $X_{212} \sim L N(8,3)$ & $Y_{112} \sim L N(650,90)$ & $Y_{212} \sim L N(860,310)$ \\
\hline
\end{tabular}


Table 3. Results of measuring the efficiency and ranking of computers

\begin{tabular}{|c|c|c|c|c|c|c|c|c|c|}
\hline$D M U$ & 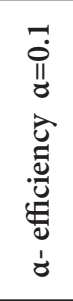 & 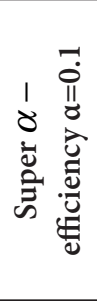 & 我 & 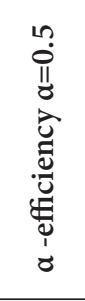 & 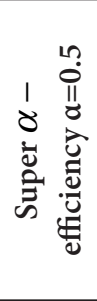 & 我 & 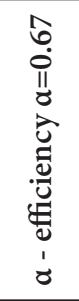 & 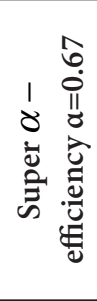 & 我 \\
\hline Acer & 1 & $e^{-78}$ & 6 & 1 & $e^{-64}$ & 4 & 1 & $e^{-59}$ & 3 \\
\hline Panasonic & 1 & $e^{-92}$ & 5 & 1 & $e^{-68}$ & 3 & 1 & $e^{-57}$ & 4 \\
\hline Sony & 1 & $e^{-420}$ & 1 & 1 & $e^{-220}$ & 2 & 1 & $e^{-160}$ & 2 \\
\hline Apple & 1 & $e^{-120}$ & 4 & $e^{68.5}$ & $e^{68.5}$ & 8 & $e^{90.3}$ & $e^{90.3}$ & 9 \\
\hline $\mathrm{Hp}$ & 1 & $e^{-70}$ & 7 & 1 & $e^{-25}$ & 5 & 1 & $e^{-3}$ & 6 \\
\hline Everex & $e^{185}$ & $e^{185}$ & 12 & $e^{226}$ & $e^{226}$ & 12 & $e^{242}$ & $e^{242}$ & 12 \\
\hline Emachines & 1 & $e^{-14}$ & 8 & $e^{72.7}$ & $e^{72.7}$ & 9 & $e^{89.7}$ & $e^{89.7}$ & 8 \\
\hline Compaq & 1 & $e^{-13}$ & 9 & $e^{24.7}$ & $e^{24.7}$ & 7 & $e^{39.2}$ & $e^{39.2}$ & 7 \\
\hline Dell & $\mathrm{e}^{151}$ & $e^{151}$ & 11 & $e^{210}$ & $e^{210}$ & 11 & $\mathrm{e}^{228}$ & $e^{228}$ & 11 \\
\hline Ibm/Lenovo & 1 & $\mathrm{e}^{-230}$ & 3 & 1 & $\mathrm{e}^{-21}$ & 6 & 1 & $\mathrm{e}^{-6.2}$ & 5 \\
\hline Toshiba & $\mathrm{e}^{95.8}$ & $\mathrm{e}^{95.8}$ & 10 & $e^{137}$ & $e^{137}$ & 10 & $e^{161}$ & $e^{161}$ & 10 \\
\hline Gateway & 1 & $e^{-380}$ & 2 & 1 & $\mathrm{e}^{-420}$ & 1 & 1 & $\mathrm{e}^{-360}$ & 1 \\
\hline
\end{tabular}

determined its rank. The above table expresses that for a set of $\alpha$ DMUs, if $\alpha<\alpha^{\prime}$, then the number of DMUs stochastic $\alpha$ - efficient is less than or equal to the number of DMUs stochastic $\alpha$-efficient and also $D M U_{o}$ is the worst DMUs for each $\alpha \in\{0.1,0.5,0.67\}$.

\section{Conclusion}

The purpose of classic MDEA model is to evaluate the performance of a set of DMUs using deterministic inputs and outputs. In real-world scenarios, stochastic models may be better suited for MDEA model when there is uncertainty associated with the inputs and/or outputs of DMUs. The stochastic inputs and outputs in MDEA model are represented with random variables. This paper attempted to extend the MDEA model under VRS technology and developed a new model with the stochastic inputs and outputs. Therefore, we proposed a stochastic output-oriented MDEA model under VRS technology for measuring the stochastic $\alpha-$ efficiency of DMUs which inputs and outputs have the log-normal distribution and obtained also a deterministic equivalent for the stochastic version. Furthermore, we proposed a stochastic super-efficiency model for ranking of
DMUs by using stochastic MDEA model. Some basic concepts in statistics were stated and the concepts of stochastic $\alpha$-efficient, $\alpha$-inefficient, super $\alpha$ - efficient, and super $\alpha$ - inefficient are defined. Finally, an example of system reliability was used to demonstrate the capability of the proposed approach. This example was run in three cases of $\alpha$ and it was observed that the number of DMUs featured stochastic $\alpha$ - inefficient and super $\alpha$ - inefficient increases when the value of $\alpha$ increases. In order to further studies, the approach of this research may be extended to some other DEA models and other distributions.

\section{References}

1. Charnes A., Cooper W.W. and Rhodes E., Measuring the efficiency of decision making units, European Journal of Operational Research, 1978;2, 429-444

2. Banker R. D. and Maindiratta A., Piecewise loglinear estimation of efficient production surfaces, Management Science, 1986;32, 126-135

3. Banker R. D., Cooper W. W., Thrall R. M., Seiford L. M. and Zhu J., Returns to scale in different DEA models, European Journal of Operational Research, 2004;154, 345-362 
4. Menger K., The laws of return: a study in meta-economics, In O. Morgenstern (Ed.) Economic activity analysis, part 111. New York: Wiley(1954)

5. Adler N., Friedman L. and Sinuany-Stern Z., Review of ranking methods in the data envelopment analysis context, European Journal of Operational Research, 2002;140, 249-265

6. Andersen P. and Petersen N. C., A procedure for ranking efficient units in data envelopment analysis, Management Science, 1993;39, 1261-1264

7. Olesen O.B. and Petersen N.C., Chance constrained efficiency evaluation, Management Science, 1995;41, 442-457
8. Cooper W.W., Huang Z.M., Lelas V., Li S.X. and Olesen O.B., Chance constrained programming formulations for stochastic characterizations of efficiency and dominance in DEA, Journal of Productivity Analysis, 1998;9, 53-79

9. Huang Z.M. and Li S.X., Dominance stochastic models in data envelopment analysis, European Journal of Operational Research, 1996;95, 370-403

10. Banker R.D., Charnes A. and Cooper W. W., Some Models for Estimating Technical and Scale Inefficiencies in Data Envelopment Analysis, Management Science, 1984;30, 1078-1092 